\title{
¿Bancos con Problemas? Un Sistema de Alerta Temprana para la Prevención de Crisis Bancarias
}

\section{Banks in trouble? A Early Warning System for the Prevention of Banking Crisis}

\author{
Ana FernándeZ-SAINZ ${ }^{1}$ \\ FELIPE LlaugeL ${ }^{2}$ \\ Universidad del País Vasco (UPV/EHU) (España) \\ Universidad Autónoma de Santo Domingo (República Dominicana)
}

Recibido el 24 de febrero de 2010 y aceptado el 27 de octubre de 2010

$\mathrm{N}^{\circ}$ de clasificación JEL: C19, C44, C61

DOI: $10.5295 /$ cdg.100239af

\section{Resumen:}

Las autoridades reguladoras y supervisoras de los sistemas financieros han probado diversos métodos para intentar encontrar un procedimiento eficaz en la elaboración de un sistema de alerta temprana de las crisis bancarias. Los Modelos de Regresión Logística han sido usados aunque han mostrado algunas debilidades, por lo que se necesitan nuevos y mejores métodos. La crisis bancaria ocurrida en la República Dominicana entre los años 2002 y 2004 se ha usado para comparar la eficacia de la Regresión Logística frente al uso del método Support Vector Machines (SVM) para la detección de crisis bancarias. En el análisis se usan 30 indicadores financieros para determinar cuáles de ellos son los más apropiados en la construcción de un modelo capaz de distinguir un banco en problemas de uno solvente. En este contexto, el método de SVM generó mejores resultados que la Regresión Logística en la detección de los bancos con problemas y se contradicen las afirmaciones de otros estudios que plantean la poca efectividad de los indicadores financieros para detectar crisis bancarias en economías emergentes.

Palabras clave:

crisis bancarias, regresión logística, support vector machines.

\footnotetext{
${ }^{1}$ Universidad del País Vasco/Euskal Herriko Unibertsitatea, Departamento de Econometría y Estadística, Avda Lehendakari Aguirre, 83, 48015 Bilbao, Spain. Email: ana.fernandez@ehu.es

${ }^{2}$ Universidad Autónoma de Santo Domingo, Departamento de Economía, República Dominicana. Email: fllaugel@ hotmail.com
}

\footnotetext{
* Agradecemos los comentarios y sugerencias de B. Sierra, S. Sperlich y de dos evaluadores anónimos. En la elaboración de este trabajo hemos contado con el apoyo financiero del proyecto UPV-038:321-13503/2001 de la Universidad del País Vasco y del proyecto SEJ2005-08269 de la Dirección General de Enseñanza Superior del Ministerio de Educación y Cultura de España.
} 


\begin{abstract}
:
The regulatory and supervisory financial authorities have tried various methods to find an effective procedure in developing an early warning system of banking crises. Logistic regression models have been used but have shown some weaknesses, so we need new and better methods. The banking crisis occurred in the Dominican Republic between 2002 and 2004 has been used to compare the effectiveness of the logistic regression method over the use of Support Vector Machines (SVM) for the detection of banking crisis. In the analysis 30 financial indicators are used to determine which ones are most appropriate to build a model able to classify banks. In this context, the SVM method produced better results than logistic regression, in detecting problem banks and contradict the findings of other studies that ask about the ineffectiveness of the financial indicators to identify banking crises in emerging economies.
\end{abstract}

\title{
Keywords:
}

banking crisis, logistic regression, support vector machines 


\section{INTRODUCCIÓN}

El objetivo de la regulación bancaria es limitar el riesgo que corren los bancos y otras entidades de intermediación financiera en sus negocios. Sin embargo, es difícil y extremadamente costoso eliminar estos riesgos, a menos que las autoridades quieran limitar severamente la propia actividad bancaria (Ahumada y Budnevich, 2001). Desde los años 80, varias crisis bancarias han ocurrido en diferentes economías, desde Asia a América Latina, y, más recientemente la crisis global iniciada en Estados Unidos ha puesto en problemas al sistema financiero mundial. El papel que juegan las autoridades monetarias y financieras es el de prevenir estas crisis con un eficiente y efectivo sistema de vigilancia. En el contexto europeo, el problema se considera de tal importancia, que la Comisión de las Comunidades Europeas, en septiembre de 2009 creó la Junta Europea de Riesgo Sistémico (JERS), que asumió la supervisión macroprudencial del sistema financiero de la Comunidad Europea, a fin de prevenir o mitigar el riesgo, de modo que se eviten episodios de perturbaciones financieras generalizadas y se garantice una contribución sostenible del sector financiero al crecimiento económico. Entre los objetivos de la JERS está el de fomentar la eficacia de los Sistemas de Alerta Temprana (SAT) mejorando la interacción entre el análisis microprudencial y el macroprudencial.

Por muchas razones, la supervisión in situ es el mejor método para evaluar la salud de un banco, pero es muy costosa en recursos (financieros, humanos y de tiempo). Dependiendo del tamaño y de las complejidades de las líneas de negocio del banco analizado, pueden pasar meses antes de que el supervisor tenga una idea precisa de la condición financiera del banco. Los SAT permiten focalizar los esfuerzos en aquellas instituciones que den alguna señal de deterioro, dejando más recursos para las evaluaciones in situ y para las inspecciones más meticulosas. Este modelo se conoce como Supervisión Basada en Riesgos (SBR).

Con el objetivo de prevenir crisis, se han desarrollado muchos modelos, usando diversos métodos, desde procedimientos ad hoc, hasta modelos basados en sofisticados análisis estadísticos. Como resultado, organizaciones internacionales y también instituciones del sector privado han empezado a desarrollar SAT, con el objetivo de anticipar cuándo una institución o un país pudiera estar afectado por una crisis financiera. El Fondo Monetario Internacional (FMI) ha liderado esfuerzos significativos en el desarrollo de SAT, macro y microprudenciales, para economías emergentes (Kaminsky y Reinhart, 1998 y Berg y Pattillo, 1999). También bancos centrales como la Reserva Federal de Estados Unidos (Kamin y Babson, 1999; Kamin et al., 2001 y King et al., 2005) y el Bundesbank (Schnatz, 1998, 1999), y académicos e instituciones del sector privado, han desarrollado modelos recientemente.

Los SAT permiten detectar debilidades y vulnerabilidades y, posiblemente, tomar medidas preventivas para reducir los riesgos de experimentar una crisis (Bussiere y Fratscher, 2002). Algunos países con gran número de bancos en su mercado han mostrado gran interés en los indicadores de alerta temprana para bancos problemáticos, ya que precisamente el elevado número de bancos hace imposible practicar supervisión in situ de forma periódica en todas las instituciones, por lo menos, una vez al año, por lo que es crucial confiar en los SAT (Ahumada y Budnevich, 2001) y en los auditores externos.

En general, gran parte de esta literatura considera sólo variables específicas de los ban$\cos$ (ratios financieros) en la estimación de un SAT, y su principal desarrollo coincide con 
un número importante de quiebras bancarias ocurridas a principios de los años 80 en países en desarrollo (Martin, 1977; Whalen y Thompson, 1989; Jones y Kuester-King, 1995; Atle y Hexeberg, 1994 y Cole, 1995). Una recopilación interesante puede ser encontrada en el trabajo de Demirgüç-Kunt (1989). Sin embargo, en general, las variables consideradas como predictivas de la insolvencia bancaria en esta literatura son variaciones de las ya mencionadas aunque se pueden considerar adicionalmente variables de rentabilidad y liquidez (Ahumada y Budnevich, 2001).

En este estudio se muestran los resultados de la comparación de dos diferentes métodos que pueden ser usados para el desarrollo de un SAT, uno, con amplia aplicación en varios países (Regresión Logística) y uno relativamente nuevo en este campo de análisis, Support Vector Machines (SVM). Ambos métodos se aplican a la información de los bancos comerciales durante la crisis bancaria ocurrida entre 2002 y 2004 en la República Dominicana. El nuevo método, Support Vector Machines, es un procedimiento procedente de la Inteligencia Computacional, desarrollado en los años 90 por Vapnik (1998).

\section{LA PREVENCIÓN DE LAS CRISIS BANCARIAS}

La mayoría de los estudios empíricos tratan de identificar los determinantes de las crisis bancarias usando datos históricos de las variables de interés de diversa periodicidad (mensual, trimestral, anual). Estos análisis se pueden realizar desde un punto de vista micro o macroeconómico. En los análisis macroeconómicos se suele encontrar que, entre los potenciales determinantes de estas crisis, están el ambiente macroeconómico, la salud del sector fiscal y el sector exterior. Sin embargo, también el desempeño del sector bancario es considerado para explicar las crisis bancarias (Wong et al., 2007). Una revisión exhaustiva de la literatura empírica se puede encontrar en Bell y Pain (2000) y en Gaytán y Johnson (2002). En González-Hermosillo et al. (1996) se toma en consideración la interacción entre variables microeconómicas, ratios financieros calculados a partir de los estados financieros de los bancos y un conjunto de factores macroeconómicos para analizar un SAT en la crisis financiera de México en 1994. Estos autores encontraron que la reducción en la actividad económica, el incremento en las tasas de interés y la depreciación de la tasa de cambio, son factores importantes para anticipar un incremento en la vulnerabilidad del sistema financiero. También los factores macroeconómicos juegan un importante rol en el momento de aparecer el problema. En particular, los autores encontraron que problemas macroeconómicos sufridos por la economía mexicana incrementaron la vulnerabilidad del sistema financiero del país. Los autores también recalcan que algunas variables relativas al sector bancario ayudaron a explicar la posibilidad de problemas, aunque no el momento de la crisis. Entre las variables específicas consideradas por estos autores está el ratio de capital ajustado por riesgo, la cartera vencida entre total de cartera, la concentración del crédito como proporción de la cartera total en ciertos sectores (tales como agricultura y vivienda), el tamaño relativo del banco y los costes operativos.

Desde un punto de vista microeconómico, Rojas-Suarez (2001) muestra que los datos individuales (a nivel de bancos) son útiles para predecir crisis bancarias empíricamente; en particular, las recientes crisis bancarias en mercados emergentes. Esto indica que las crisis bancarias pudieran ser también reveladas a través de la vigilancia puntual del sector 
bancario (Wong et al., 2007). Demirgüc-Kunt y Detragiache (1998) usaron modelos logit y probit para estudiar los determinantes de las crisis bancarias a partir de datos anuales de 65 economías desarrolladas y en desarrollo en el período 1980-1994. Las variables explicativas consideradas fueron clasificadas en cuatro grupos: variables macroeconómicas, variables financieras, variables institucionales y variables de pasadas crisis. Los resultados empíricos indican que las crisis bancarias estaban asociadas, principalmente, con el ambiente macroeconómico de bajo crecimiento, alta inflación y altas tasas de interés real. Además, la situación de la balanza de pagos se encontró asociada a problemas bancarios sistémicos. Sin embargo, hay que tener en cuenta que no considerar los 'efectos de contagio' a través de los países y el uso de datos de baja frecuencia son una de las mayores debilidades de ese procedimiento econométrico (Wong et al., 2007). En Martin (1977) se desarrolla un modelo SAT para determinar la solidez del sistema bancario, expresando la probabilidad de una crisis futura en función de variables obtenidas del balance general y del estado de resultados del período actual. El modelo se estima en base a la información financiera de crisis bancarias anteriores y de las calificaciones de auditores bancarios.

Trabajos más recientes dan cuenta de los diferentes métodos empleados por las autoridades de supervisión financiera en el desarrollo de SAT. Sahajwala y Bergh (2000) hacen un repaso a los métodos usados por países del G10. Ellos describen los sistemas desarrollados y en proceso de implementación desde los años 90, señalando algunos cuyo uso fue discontinuo. En su trabajo Sahajwala y Bergh (2000) agrupan los SAT en cuatro tipos: a) Sistema de calificación de supervisores bancarios; b) Sistema de análisis de pares y razones financieras; c) Sistema de evaluación de riesgos bancarios y d) Modelos estadísticos. Además, destacan la superioridad de los métodos estadísticos para detectar bancos en problemas, principalmente por la minimización del error tipo I (fallo en la detección de bancos en problemas) con respecto al error tipo II (señalar un banco viable como banco fallido).

\section{RECONOCIMIENTO DE PATRONES E INDICADORES FINANCIEROS PARA LA EVALUACIÓN DE BANCOS}

El análisis de instituciones de intermediación financiera a partir de indicadores calculados en base a los estados financieros no es nuevo. Muchos estudios han demostrado que el análisis de los indicadores, de forma individual, puede ser engañoso, especialmente cuando lo que se busca es determinar un umbral dentro del cual cada indicador debe estar ubicado y que, en cuanto sobrepase dicho umbral, se genere una señal de alerta. Esto implica el desarrollo de una metodología que analice, no los indicadores de forma individual, sino al patrón que muestra el conjunto de indicadores de relevancia.

En Rojas Suarez (2001) se muestra que algunos de los indicadores más utilizados en los países industrializados, como la relación capital-activo, tienen un pobre comportamiento como indicador de problemas en América Latina y Asia del Este. Así, se sostiene que los indicadores CAMELS (Capital, Asset Quality, Management, Liquidity, Earnings, Sensibility), instaurados en 1979 por las agencias reguladoras federales de los Estados Unidos para evaluar entidades, pueden no ser efectivos para reflejar la condición financiera de un banco en economías emergentes, debido a posibles problemas contables (Montas, 2009). Otros autores (Hardy y Pazarbasioglu, 1999) evalúan el uso de variables macroeconómicas 
para la detección temprana de crisis bancarias, obteniendo resultados interesantes, pero no concluyentes, ya que el modelo que proponen para detectar crisis se aplica a todo el sector financiero de Asia y no a un banco en particular.

Este estudio está enfocado al desarrollo de un modelo que permita a las autoridades supervisoras del sistema financiero detectar con suficiente antelación, señales de problemas en los bancos de manera que permita tomar acciones correctoras para prever problemas. El modelo que se propone está basado en indicadores calculados a partir de las informaciones de los estados financieros de los propios bancos.

El reconocimiento de patrones, en general, consiste en asignar un objeto a cada una de las categorías o clases. Para un problema específico de reconocimiento de patrones, un clasificador es un método que clasifica los objetos correctamente en sus clases con razonable precisión (Abe, 2005). En el problema de interés en este estudio (clasificación de bancos) se consideran dos clases: la clase 1 corresponde a los bancos con problemas financieros y la clase 2 a los bancos financieramente viables.

El procedimiento Support Vector Machines (SVM) fue desarrollado por Vapnik en los años 90 del siglo pasado (Cristianini y Shawe-Taylor, 2000). Recientemente, Yi et al. (2007) han probado la efectividad de estos modelos en la evaluación de corporaciones. De acuerdo a este estudio, SVM superó las debilidades de otros modelos como los modelos lineales y las redes neuronales. En el mismo sentido, Shu-Xia y Wang (2004) hacen la comparación entre cuatro métodos de clasificación (Lagrangian Support Vector Machine (LSVM), Finite Newton Lagrangian Support Vector Machine (NLSVM), Smooth Support Vector Machine (SSVM) y Finite Newton Support Vector Machine (NSVM)). El estudio concluye con una comparación de la capacidad de esos algoritmos para generar clasificadores, lineales o no lineales, y proporciona pautas para seleccionar el más apropiado de los cuatro métodos de clasificación en un problema concreto. En este contexto, los resultados de Mangasarian y Musicant (2001) y de Yi et al. (2007) han sido usados, en este trabajo, para construir y probar un modelo SVM básico basado en ratios financieros de bancos comerciales.

La Superintendencia de Bancos de la Republica Dominicana, al igual que otros bancos centrales, utiliza muchos indicadores para evaluar las instituciones financieras. Entre ellos, el más utilizado es el índice de solvencia, que mide el grado de cobertura de riesgos en que incurre una institución en función del capital de la misma y que durante el período de la crisis estaba estipulado en un mínimo de 10\%. Montas (2009) señala que dicho indicador resultó poco efectivo para detectar la condición deficitaria de los bancos que posteriormente colapsaron. Montas muestra cómo, desde 1998 al 2002 el índice de solvencia ponderado por riesgo de los tres bancos que posteriormente quebraron (Baninter, Bancredito y Mercantil) fue superior al de los bancos principales del sistema que mostraron con el tiempo ser bancos saludables (Reservas, Popular, BHD, Citi Bank, Scotia Bank y Progreso). Un resultado similar fue encontrado en otros indicadores: Provisión de cartera/cartera vencida, ROE y ROA. Un tercer grupo de indicadores, como Gastos Financieros/activos productivos promedio, mostraban un comportamiento indistinto para ambos grupos de bancos. Una de las conclusiones del trabajo de Montas (2009) es que:

"Se puede establecer que los indicadores financieros y bancarios que se han utilizado usualmente hasta ahora para monitorear el comportamiento del sistema financiero dominicano no constituyen un instrumento eficaz para evaluar y alertar sobre la verdadera 
situación de las entidades financieras en un entorno macroeconómico cambiante. Los indicadores deben ser más consistentes para observar cambios en el corto plazo".

En este estudio obtenemos que los indicadores usados no son malos, sino que el problema está en el modo en el que se han empleado para la evaluación de bancos. En el mismo sentido, Kaminsky y Reinhart (1999) examinaron el comportamiento de 15 indicadores macroeconómicos para una muestra de 20 países que experimentaron una crisis bancaria durante el período 1970-1995. El comportamiento de los indicadores durante los dos años anteriores a la crisis fue contrastado con su comportamiento en épocas 'normales'. De esta forma, se considera que el indicador emite una señal, si es que traspasa cierto valor umbral, siendo este umbral el que minimiza el número de falsas alarmas (Montas, 2009). Posteriormente, Kaminsky (1999) propuso desarrollar un indicador compuesto, que aglutinara la información de múltiples variables similares a las empleadas en 1996. Sin embargo, los resultados preliminares muestran que estos indicadores tienen peores resultados que los indicadores individuales (Montas, 2009). Estos resultados fortalecen la hipótesis de este estudio, en el sentido de que, no son los indicadores individuales los que deben ser analizados, sino el patrón de comportamiento que presentan los mismos.

Los datos con los que se ha realizado el análisis provienen de los estados financieros mensuales de 14 bancos comerciales de la República Dominicana en el período noviembre 2002 - diciembre 2004. A partir de dicha información se construyeron 30 indicadores financieros para cada uno de los bancos. Los datos abarcan el período correspondiente a la crisis financiera, periodo en el que colapsaron tres importantes bancos. La importancia de este período es que se produjo la crisis financiera más importante de la República Dominicana. El banco más grande del sistema financiero colapso, así como también otros dos de mediano tamaño desde el punto de vista del volumen de activos. Además, para este periodo, se tiene abundante información ex post del comportamiento de esos bancos, lo que permite definir claramente cuando dichos bancos comenzaron a manifestar señales claras de deterioro.

Las técnicas estadísticas de análisis multivariante han sido empleadas para predecir quiebras de compañías, basándose en los estados financieros (Altman, 1968). Algunos trabajos (Todorov et al., 1999) indican que la función de clasificación se puede construir con un número relativamente reducido de indicadores, debido a la gran correlación que comúnmente se observa entre ellos. En el Anexo 1 se muestra cómo fueron construidos los indicadores y en la Tabla 1, a continuación, se muestra la agrupación realizada.

Tabla 1

\section{Grupos de Indicadores Financieros}

\begin{tabular}{|c|c|}
\hline Grupo & Indicadores \\
\hline Estructura de Activos & $\mathrm{I} 1, \mathrm{I} 2, \mathrm{I} 3, \mathrm{I} 4, \mathrm{I} 5, \mathrm{I} \mathbf{6}, \mathrm{I} 7, \mathbf{I 1 9}, \mathbf{I 2 0}$ \\
\hline Patrimonio & I8,I9,I10,I11 \\
\hline Cartera de préstamos & $\mathrm{I} 12, \mathrm{I} 13, \mathrm{I14}, \mathrm{I} 15, \mathrm{I} 16$ \\
\hline Liquidez & I17,I18 \\
\hline Eficiencia Administrativa & $\mathrm{I} 21, \mathbf{I 2 2}, \mathrm{I} 23, \mathbf{I 2 4}, \mathrm{I} 28$ \\
\hline Otros & $\mathrm{I} 25, \mathrm{I} 26, \mathrm{I} 27, \mathrm{I} 29, \mathrm{I} 30$ \\
\hline
\end{tabular}


Se analizaron los 30 indicadores para seleccionar los más apropiados en la función discriminante. En cada paso se selecciona aquel indicador que tiene la capacidad discriminante más alta (el valor de lambda de Wilks más pequeño). En base a este procedimiento, los indicadores seleccionados fueros: I6, I8, I10, I14, I17, I19, I20, I22 e I24. Merece la pena resaltar que tres de ellos (I6, I19 y I20) son indicadores de la estructura de activos, I14 es un indicador de cartera, I8 y I10 son indicadores de patrimonio, I22 y I24 son indicadores de eficiencia administrativa y $\mathrm{I} 17$ es un indicador de liquidez. En conjunto, los indicadores seleccionados incluyen todos los aspectos principales de la evaluación de bancos y son los indicadores comúnmente utilizados por las superintendencias de bancos y bancos centrales de los países.

\section{METODOLOGÍA SUPPORT VECTOR MACHINES}

La metodología conocida como Support Vector Machines consiste básicamente, en modelos matemáticos de optimización, desarrollados para implementar clasificación de patrones. SVM es un método, relativamente nuevo, de clasificación de patrones, enmarcado en el campo no paramétrico. Otros métodos no paramétricos son las redes neuronales y los sistemas difusos. Todos estos métodos se desarrollan usando pares de entrada-salida para el entrenamiento de los mismos. El clasificador adquiere la facultad de distinguir entre diferentes patrones a través del desarrollo de funciones de decisión (Abe, 2005). Entrenar un clasificador implica maximizar el desempeño de su tarea usando datos de entrenamiento. La habilidad de la generalización pierde efectividad si el clasificador se ajusta exactamente a los datos de entrenamiento. Por lo tanto, es muy importante desarrollar el método apropiado para el proceso de entrenamiento y, así, obtener buenas características de clasificación.

Para un problema de dos clases, un SVM se entrena de manera que la función de decisión maximize la habilidad de generalización. Esta situación se muestra en la Figura 1 , donde se trata de encontrar una forma de separar los bancos solventes de los bancos fallidos. En dicha Figura se muestra la situación si se usaran sólo dos indicadores $\left(\chi_{1}\right.$ y $\left.\chi_{2}\right)$.

Las ventajas del SVM sobre otros métodos de clasificación ya ha sido evaluada (Min y Lee, 2005). En ese estudio se comparó el desempeño de los métodos Logit, Redes Neuronales, Análisis Discriminante Múltiple y SVM, y se demostró, con datos empíricos de empresas de Corea, que el método de SVM es superior a los demás, tanto en velocidad de desarrollo, como en ajuste de los parámetros para la predicción de una bancarrota. 
Figura 1

Hiperplano de separación optima en espacio de dos dimensiones

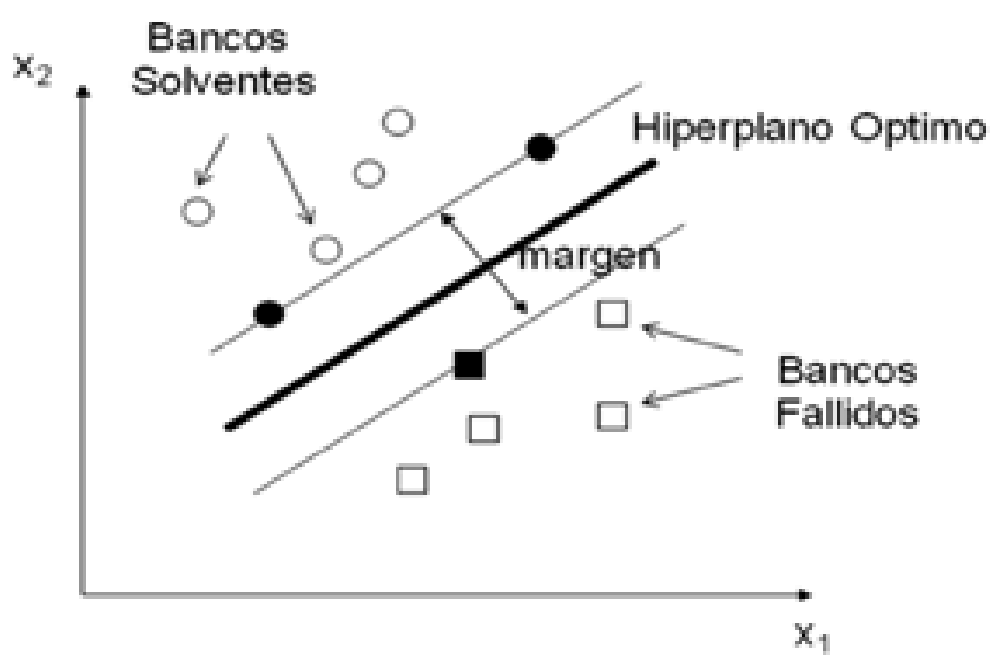

La habilidad de generalización depende, fundamentalemnte, de la localización del hiperplano de separación y para determinar este hiperplano de separación óptimo usamos la distancia euclidiana entre un dato de entrenamiento x y el hiperplano de separación. Todo dato de entrenamiento debe satisfacer la condición de tener una distancia al hiperplano, mayor que la distancia de los vectores de soporte.

El criterio de clasificación es el siguiente: clase 1 (banco viable), si $\mathrm{D}(\mathrm{x})>0$; clase 2 (banco en problemas), si $\mathrm{D}(\mathrm{x})<0$. Si $\mathrm{D}(\mathrm{x})=0$ el conjunto de indicadores no es clasificable. $\mathrm{D}(\mathrm{x})$ es la función de decisión o clasificación.

\section{CLASIFICADOR DE INDICADORES FINANCIEROS}

La información compuesta por los 9 indicadores seleccionados para los 14 bancos comerciales en el periodo noviembre 2002-diciembre $2005^{1}$ fue usada para implementar la clasificación; así, se dispone de 420 casos; a cada caso se le asigna una etiqueta para indicar el estatus del banco en el mes correspondiente ${ }^{2}$, es decir, cada caso es un vector fila con 10 columnas, que se corresponden con 9 indicadores y una etiqueta.

En toda la base de datos, sólo 20 casos corresponden a bancos colapsados y ésto es un grave inconveniente para muchos algoritmos de reconocimiento de patrones. En general, se recomienda tener el mismo número de ejemplos en ambos casos. Sin embargo, como

\footnotetext{
${ }^{1}$ Este período de tiempo incluye 12 meses posteriores a la crisis bancaria.

${ }^{2} 0$ para bancos sin problemas, y 1 para bancos en crisis o colapsados.
} 
mostraremos en los resultados, en este análisis ésto no es un problema. Para realizar el cálculo, se separan aleatoriamente los datos suministrados en dos conjuntos, uno para el entrenamiento (con $50 \%$ de los datos) y otro para la prueba (con el restante 50\%).

Como índice de clasificación se usa la proporción de casos correctamente clasificados.

Recuérdese que se les asignó un estatus 0 a los bancos viables y 1 a los no viables. Si $D_{k}$ representa el estatus real de cada banco en el mes $\mathrm{k} \mathrm{y} \hat{D}_{k}$, el estatus predicho por el modelo, donde $D_{k} \in(0,1)$, entonces, el índice de clasificación será:

$$
\gamma_{D}=1-\frac{1}{N} \sum_{k=1}^{N}\left(\hat{D_{k}}-D_{k}\right)^{2}
$$

donde $0 \leq \gamma_{D} \leq 1$ y $\mathrm{N}$ es el número de casos.

Para tener un estimador más preciso del índice de clasificación, se ejecutaron 1000 pruebas, en la Figura 2 se muestra el histograma del índice de clasificación. El valor mínimo del índice fue 0.9143, el máximo 1.0000. En el 75\% de las pruebas el índice de clasificación fue superior a 0.9714 . La mediana tomo el valor 0.9810 y la media 0.9786 . Como se puede observar, el índice de clasificación no está simétricamente distribuido, con un sesgo notable hacia la derecha.

Figura 2

\section{Histograma del índice de clasificación después de 1000 pruebas}

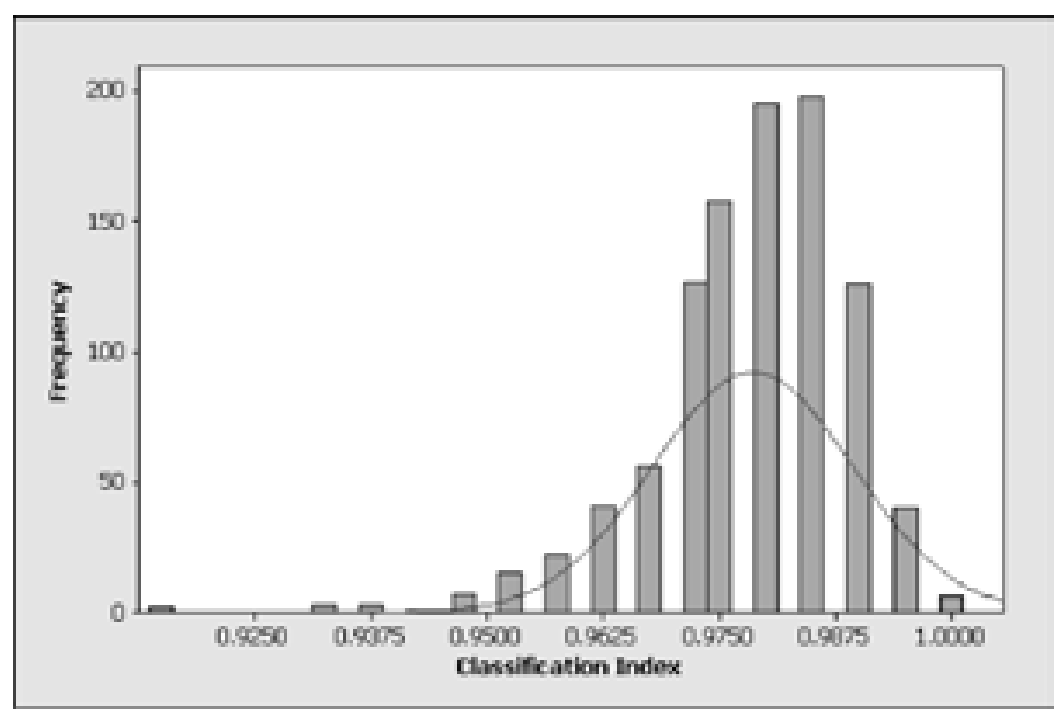


La Figura 3 muestra que en la mayoría de las pruebas el índice de clasificación fue muy bueno y en 4 ocasiones se obtuvo un $100 \%$ de precisión en la clasificación. Este es un resultado muy importante, dada, como ya se ha dicho, la mala conformación de los datos de entrenamiento, donde había muy pocos ejemplos de bancos fallidos y aún así, el modelo fue capaz de reconocerlos. La distribución de los índices de clasificación también indica que no se observó el problema de sobreentrenamiento.

Figura 3

\section{Índice de clasificación por número de prueba}

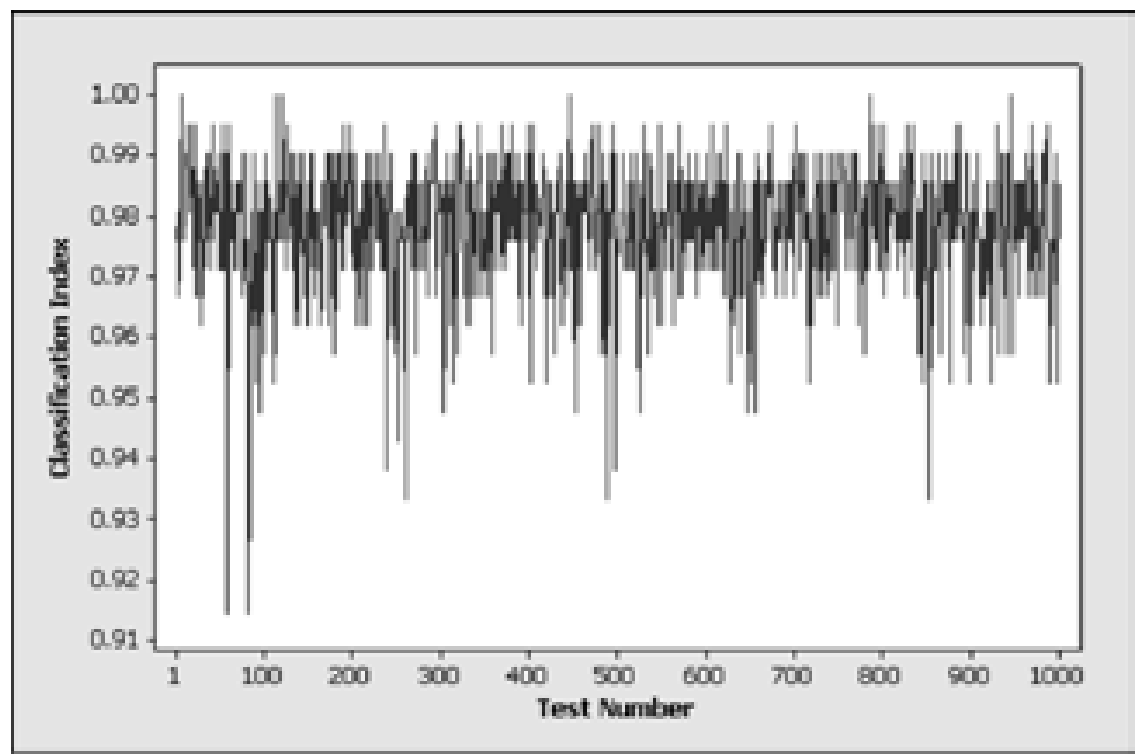

En los anexos se muestra la distribución de los indicadores, en conjuntos de dos y tres indicadores, siendo notoria la imposibilidad de separar los bancos sin problemas de los bancos fallidos con un hiperplano (no separable linealmente). Los gráficos aconsejan el uso de algún tipo de ajuste no lineal, o el uso de una transformación tipo kernel.

Para verificar el desempeño del modelo para separar los bancos fallidos de los viables, se hizo el mismo experimento, usando los indicadores del período Enero 2002 a Diciembre 2004, es decir, 10 meses antes de la crisis, más el periodo de la crisis. El índice de clasificación tuvo un mínimo de 0.9052 y un máximo de 0.9905 , con una media de 0.9567 . La Figura 4 muestra el histograma y el índice de clasificación por número de prueba. Esta última prueba se hizo para probar que el modelo era bueno tomando meses previos a la crisis también. En la Figura 4 se muestran los resultados. 
Figura 4

Histograma e Índice de clasificación por número de prueba. Datos de Enero 2002 a Diciembre 2004

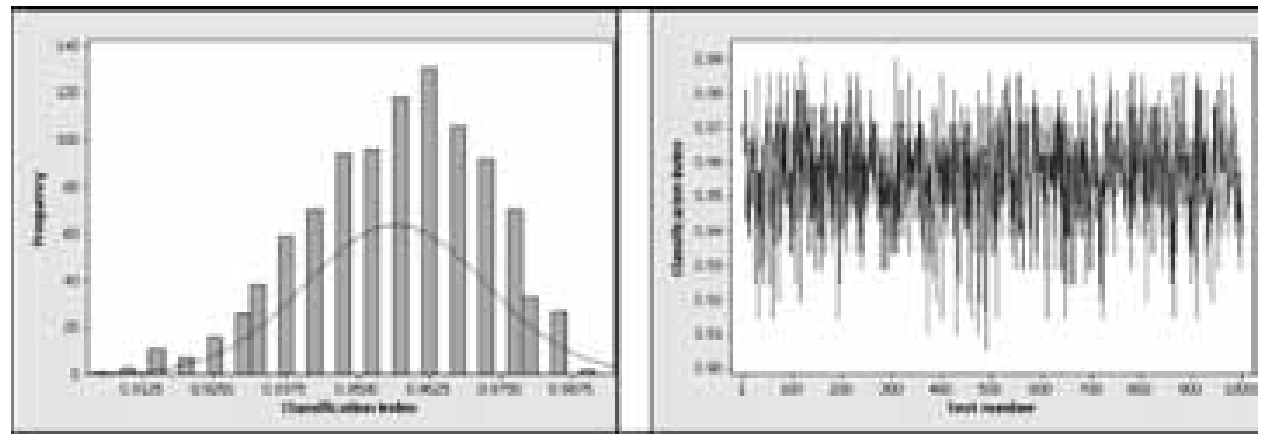

\section{COMPARACIÓN DE DESEMPEÑO}

La información de los indicadores propuestos en el período de 30 meses considerado se ha usado para desarrollar un modelo de regresión logística para detectar bancos con problemas. Estos datos fueron divididos en dos subconjuntos; uno, para ajustar el modelo y otro, para probarlo. El conjunto para el ajuste del modelo se formó con los indicadores del período noviembre 2002-diciembre 2004, período durante el cual hubo una crisis bancaria. El conjunto de prueba se formó con datos previos (enero 2002-octubre 2002) y posteriores a la crisis (enero 2005-diciembre 2005). Así, el modelo logit considerado es:

$$
\pi(\mathrm{x})=\frac{e^{g(x)}}{1+e^{g(x)}} \quad \text { donde } \mathrm{g}(\mathrm{x})=\beta_{0}+\beta_{1} x_{1}+\beta_{2} x_{2} \ldots+\beta_{p} x_{p}
$$

y $\left(x_{1}, x_{2}, \ldots, x_{p}\right)$ representan los indicadores considerados.

La estimación del modelo, teniendo en cuenta únicamente los indicadores estadísticamente significativos, es:

Tabla 2

Ajuste del modelo Logit

\begin{tabular}{lrrrrrrrrr}
\hline \multirow{2}{*}{ Variables } & \multicolumn{1}{c}{ B } & E.T. & Wald & gl & Sig. & Exp(B) & \multicolumn{2}{c}{ I.C. para EXP(B) } \\
\cline { 2 - 9 } & & & & & & & Inferior & Superior \\
\hline I7 & 1.242 & .683 & 3.305 & 1 & .069 & 3.462 & .908 & 13.206 \\
I19 & -2.194 & .716 & 9.390 & 1 & .002 & .111 & .027 & .453 \\
I25 & -2.700 & 1.070 & 6.367 & 1 & .012 & .067 & .008 & .547 \\
Constante & 165.709 & 54.752 & 9.160 & 1 & .002 & $9.26138720 \mathrm{E}+71$ & & \\
\hline \multicolumn{2}{c}{$\mathrm{g}(\mathrm{x})=165.709+1.242 * \mathbf{I 7}-2.194 * \mathbf{I 1 9}-2.7 * \mathbf{I 2 5}$} \\
\end{tabular}


Posteriormente y con el objeto de mejorar el desempeño del modelo logit, se procedió a usar bootstrap (Llaugel 2008).

Los índices de clasificación para el modelo en los períodos de prueba fueron 0.7040 y 0.9512 , respectivamente, en el caso de la Regresión logística. El mismo índice para el clasificador SVM en el mismo periodo de prueba, fue en promedio 0.9786.

Tabla 3

Índice de Clasificación de los modelos Logit vs SVM

\begin{tabular}{lccc}
\hline \multirow{2}{*}{ Periodos de prueba } & Logit & $\begin{array}{l}\text { Logit con } \\
\text { Bootstrap }\end{array}$ & SVM \\
\cline { 2 - 4 } & 0.7040 & 0.7200 & 0.9567 \\
\hline Enero 2002- Octubre 2002 & 0.9512 & 0.9512 & 0.9786 \\
\hline Enero 2005- Diciembre 2005 & & \\
\hline
\end{tabular}

Aunque la Tabla 3 muestra una mejoría importante en el índice de clasificación usando SVM, hay que tener en cuenta también los errores tipo I y tipo II para los tres modelos. Como el objetivo de este estudio es comparar la bondad de los modelos para detectar crisis bancarias, a fin de construir un sistema de alerta temprana, el poder de predicción del modelo es crucial. Un método convencional para evaluar este poder predictivo es construir una tabla de contingencia de dos vías, donde se colocan los resultados de la clasificación en una matriz dos por dos (Kaminsky y Reinhart, 1999).

La Tabla 4 resume la tabla de contingencia para los dos modelos, Regresión Logística, considerando bootstrap y no haciendolo y Support Vector Machines. El error tipo I es la probabilidad de no detectar señales de alarma cuando hay razones para ello y el error tipo II es la probabilidad de producir señales de alerta cuando no hay condiciones que lo indiquen (falsa alarma).

Tabla 4

Porcentaje de clasificación correcta Logit vs $\mathrm{SVM}^{3}$

\begin{tabular}{|c|c|c|c|c|c|c|c|}
\hline \multirow{3}{*}{$\begin{array}{l}\text { Periodo de } \\
\text { prueba }\end{array}$} & \multirow{3}{*}{$\begin{array}{c}\text { Estatus } \\
\text { Real }\end{array}$} & \multicolumn{4}{|c|}{ Regresión Logistica } & \multirow{2}{*}{\multicolumn{2}{|c|}{$\begin{array}{l}\text { Support Vector } \\
\text { Machines }\end{array}$}} \\
\hline & & \multicolumn{2}{|c|}{ Original } & \multicolumn{2}{|c|}{ Bootstrapping } & & \\
\hline & & 0 & 1 & 0 & 1 & 0 & 1 \\
\hline \multirow{2}{*}{$\begin{array}{l}\text { Ene-Oct } \\
2002\end{array}$} & 0 & $72.63 \%$ & $27.37 \%$ & $84.21 \%$ & $15.79 \%$ & $91.40 \%$ & $8.60 \%$ \\
\hline & 1 & $36.67 \%$ & $63.33 \%$ & $66.67 \%$ & $33.33 \%$ & $4.00 \%$ & $96.00 \%$ \\
\hline \multirow{2}{*}{$\begin{array}{l}\text { Ene-Dic } \\
2005\end{array}$} & 0 & $99.11 \%$ & $0.89 \%$ & $100.00 \%$ & $0.00 \%$ & $98.50 \%$ & $1.50 \%$ \\
\hline & 1 & $36.36 \%$ & $63.64 \%$ & $\mathbf{5 4 . 5 5 \%}$ & $45.45 \%$ & $0.00 \%$ & $100.00 \%$ \\
\hline
\end{tabular}

${ }^{3}$ el error tipo I en negrita y el error tipo II en itálica. 
En la Tabla 4 se puede ver la superioridad del modelo SVM con respecto al logit. En el primer período de prueba (Enero - Octubre de 2002), mientras el modelo original logit produjo $27.37 \%$ de falsas alarmas, SVM sólo produjo un $8.60 \%$. Los bancos en problemas sólo fueron detectados en un $63.33 \%$ de las veces con el modelo logit, en contraste con el modelo SVM que acertó en el $96.00 \%$ de los casos. Los resultados para el segundo período de prueba (Enero - Diciembre de 2005) fueron aún mejores; el modelo logit decreció en falsas alarmas a $0.89 \%$ y con SVM fue $1.5 \%$, pero el porcentaje de bancos en problemas detectado por el modelo logit mejoró muy poco (63.64\%), mientras que el modelo SVM detectó el 100\% de los bancos con problemas. La mejoría mostrada por la regresión logística cuando se considera bootstrap fue sólo la reducción de falsas alarmas (15.79 y $0.00 \%$ respectivamente), pero la detección de bancos fallidos fue peor que con el modelo logit original en los dos periodos, $33.39 \%$ y $45.45 \%$, respectivamente.

\section{CONCLUSIONES}

Sin duda, un Sistema de Alerta Temprana (SAT) para detectar bancos en problemas puede ser una herramienta muy importante para las autoridades supervisoras de los sistemas financieros de los países. En general, la principal preocupación de las autoridades supervisoras es poder realizar una supervisión basada en riesgos, ya que, de esta forma, se hace un uso óptimo de los escasos recursos humanos y financieros. El aporte principal de esta investigación es proveer una nueva herramienta para el desarrollo de un Sistema de Alerta Temprana que pueda evaluar rápidamente la abundante información financiera que proviene de los bancos y detectar con tiempo la posibilidad de problemas en un banco en particular y, así, tomar medidas preventivas, si fuese necesario.

Los sistemas de alerta basados en modelos estadísticos han probado ser proactivos en la detección de señales de deterioro en la condición financiera de los bancos. Uno de los mejores SAT estadísticos es la regresión logística. En el análisis realizado en este trabajo, este método produce buenos resultados cuando se prueba con datos de la crisis financiera de la República Dominicana, ocurrida entre los años 2002 y 2004. En este caso, la regresión logística precisa de pocos indicadores relevantes. Aunque esos indicadores cubren los principales aspectos del negocio bancario, en ocasiones puede ser preferible considerar un espectro más amplio de variables. En el presente estudio se propone el uso del método Support Vector Machines para comparar la eficiencia del modelo logit en la detección de bancos con problemas. Así, a partir de los datos iniciales, se construye un subconjunto de datos para ajustar el modelo propuesto y otro para la prueba y se usa un índice de clasificación para evaluar la bondad del modelo, en periodos previos y posteriores a la crisis. Dicho índice toma un valor de 0.7040 y 0.9512 , respectivamente, el cual fue mejorado usando las técnicas de bootstrap. Los resultados obtenidos indican que el modelo Logit no es capaz de identificar apropiadamente los bancos a punto de fallar en el periodo previo a la crisis, aunque mejora su desempeño después de la crisis. Por otro lado, el modelo Support Vector Machines supera a la regresión logística en los dos periodos de prueba. El índice de clasificación para este modelo es de 0.9567 y 0.9786 respectivamente en los periodos de prueba. También hay que resaltar que esta aproximación presenta menos señales de falsas alarmas (error Tipo II) y detecta a los bancos con problemas en casi el 100\%. Es importante señalar 
que, de incorporarse mayor información al modelo, bien con un periodo de análisis más amplio, ó bien con información no pública que sólo maneja la Superintendencia de Bancos, el desempeño del modelo pudiera ser muy superior.

El objetivo de esta investigación ha sido construir un SAT dinámico capaz de ser reentrenado con nueva información, sin olvidar la anterior. Los métodos basados en SVM pueden ser reentrenados cuando se detectan nuevos patrones en el comportamiento de los indicadores de los bancos y, de esta manera, el modelo mejorará su capacidad de reconocimiento de los posibles patrones de deterioro presentes en el sistema financiero. Este último aspecto es muy importante si se toma en cuenta lo que algunos investigadores señalan sobre la dinámica de las crisis bancarias (King et al., 2005). En general, los SAT deben ser muy flexibles para poder adaptarse a los cambios, a veces muy rápidos, que presenta el sistema financiero.

\section{REFERENCIAS BIBLIOGRAFICAS}

ABE, S. (2005): Support Vector Machines for Pattern Classification. Springer-Verlag London Limited. AHUMADA, A. Y BUDNEVICH, C. (2001): "Some Measures of Financial Fragility in the Chilean Banking System: an Early Warning Indicator Application”. Banco Central de Chile, Working Paper \# 117.

ALTMAN, E.I. (1968): "Financial ratios, discriminant analysis and the prediction of corporate failure". Journal of Finance.

ATLE B.S., Y HEXEBERG, B. (1994): "Early Warning Indicators for Norwegian Banks: A Logit Analysis of the Experiences from the Banking Crisis". Oslo, Norway: Norges Bank Research Department.

BELL, J. Y PAIN, D. (2000): "Leading Indicator Models of Banking Crises: a Critical Review". Financial Stability Review, Bank of England.

BERG, A. Y PATTILLO, C. (1999): "Predicting Currency Crises: The Indicators Approach and an Alternative". Journal of International Money and Finance.

BUSSIERE, M. Y FRATZSCHER, M. (2002): “Toward a new Early Warning System of Financial Crises". European Central Bank. Working Paper No. 145.

COLE, R. A. (1995): "FIMS: A New Monitoring System for Banking Institutions". Federal Reserve Bulletin.

CRISTIANINI, N. Y SHAWE-TAYLOR, J. (2000): “An Introduction to Support Vector Machines and other Kernel-based Learning Methods". Cambridge University Press. Cambridge. UK.

DEMIRGÜC-KUNT, A. (1989). "Deposit-Institution Failures: A Review of Empirical Literature." Federal Reserve Bank of Cleveland, Economic Review.

DEMIRGÜC-KUNT, A., Y DETRAGIACHE, E. (1998): “The Determinants of Banking Crises in Developing and Developed Countries". International Monetary Fund Staff Paper.

FONDO MONETARIO INTERNACIONAL (2006): Indicadores de solidez financiera. Guía de Compilación.

GAYTÁN, A. Y JOHNSON, C.A. (2002): "A Review of the Literature on Early Warning Systems for Banking Crises". Working Paper 183, Central Bank of Chile.

GONZÁLEZ-HERMOSILLO, B.; PAZARBASIOGLU, C. Y BILLINGS, R. (1996): “Banking System Fragility: Likelihood versus Timing of Failure: An Application to the Mexican Financial Crisis". IMF Working Paper. Washington: International Monetary Fund

HARDY, D. Y PAZARBASIOGLU, C. (1999): "Determinant and Leading Indicators of Banking Crisis: Further Evidence”. International Monetary Fund Staff Papers. 
JONES, D. S. Y KUESTER-KING, K. (1995): “The Implementation of Prompt Corrective Action: An Assessment". Journal of Banking and Finance.

KAMIN, S. Y BABSON, O. (1999): "The Contribution of Domestic and External Factors to Latin American Devaluation Crises: An Early Warning Systems Approach”, Board of Governors of the Federal Reserve System, International Finance Discussion Papers.

KAMIN, S.; SCHINDLER, J. Y SAMUEL, S. (2001): "The Contribution of Domestic and External Factors to Emerging Market Devaluation Crises: An Early Warning Systems Approach”, International Finance Working Paper, Board of Governors of the Federal Reserve System.

KAMINSKY, G. (1999): "Currency and Banking Crises: The Early Warning of Distress". IMF Working Paper.

KAMINSKY, G. Y REINHART, C.M. (1998): "Financial Crises in Asia and Latin America: Then and Now". American Economic Review, Papers and Proceedings.

KAMINSKY, G. Y REINHART, C.M. (1999): "The Twin Crises: the Causes of Banking and Balance-of-payments Problems". American Economic Review.

KING, T.; NUXOLL, D. Y YEAGER, T. (2005): "Are the Causes of Banking Distress Changing? Can Researchers Keep Up?”. FDIC Center for Financial Research Working Paper.

LLAUGEL, F. 2008. "Improving Logit Models using Bootstrapping: A Banking Crisis Prevention Application". Mimeo.

MANGASARIAN, O. L. Y MUSICANT, D. R. (2001): "Lagrangian Support Vector Machines". Journal of Machine Learning Research.

MARTIN, D. (1977): "Early Warning of Bank Failure: A Logit Regression Approach". Journal of Banking and Finance.

MIN, J. Y LEE, Y. (2005): "Bankruptcy Prediction using Support Vector Machine with optimal choice of kernel function parameters". Expert Systems and Applications.

MONTAS, J.T. (2009): La Crisis Bancaria del 2003, Como y por qué. Editora Alfa y Omega CxA. Santo Domingo.

ROJAS-SUAREZ, L. (2001): "Rating Banks in Emerging Markets: What Credit Rating Agencies Should Learn from Financial Indicators". Working Paper, Peterson Institute for International Economics.

SAHAJWALA, R. Y VAN DEN BERGH, P. (2000): "Supervisory Risk Assessment and Early Warning System". Basel Committee on Banking Supervision Working Papers.

SCHNATZ, B. (1998): "Macroeconomic Determinants of currency turbulences in emerging markets". Discussion paper, Economic Research Group of the Deutsche Bundesbank.

SCHNATZ, B. (1999): "The Sudden Freeze of the Asian Miracle: The Role of Macroeconomic Fundamentals". Asia Pacific Journal of Finance.

SHU-XIA, L. Y WANG, X. (2004): “A Comparison among four SVM Classification methods: LSVM, NLSVM, SSVM and NSVM". Proceeding of the Third International Conference on Machine Learning on Cybernetics.

TODOROV, V.K, NEYKOV, N.M., AND NEYTCHEV, R.V. (1999). Robust Selection of Variables in the Discriminant Analysis based on MVE and MCD estimators. Proceedings of International Computational Statistics $9^{\text {th }}$ Symp. 193-198.

VAPNIK, N.V. (1998): Statistical Learning Theory. John Wiley and Sons, Inc. New York.

WHALEN, G. Y THOMPSON, J.B. (1989): "Using Financial Data to Identify Changes in Bank Condition". Federal Reserve Bank of Cleveland Economic Review.

WONG, J.; WONG, E. Y LEUNG, P. (2007): "A Leading Indicator Model of Banking Distress an Early Warning System for Hong Kong and other EMEAP economies". Hong Kong Monetary Authority, Working Paper.

YI, P. L.; QIU, D. F. Y BAO, P. (2007): “A Study of Financial Distress Prediction of Listed Corporations with Support Vector Machines Model". Proceedings of 2007 IEEE International Conference on Grey Systems and Intelligent Services. 


\section{Anexo 1: DEFINICIÓN DE INDICADORES FINANCIEROS}

\begin{tabular}{|c|c|c|c|}
\hline Indicadores & Código & Indicadores & Código \\
\hline Disponibilidades/Activos & I1 & $\begin{array}{l}\text { Provisión para Cartera de Créditos/ } \\
\text { Cartera de Créditos }\end{array}$ & I16 \\
\hline Cartera de Créditos/Activos & $\mathrm{I} 2$ & Disponibilidades/Total de Captaciones & I17 \\
\hline Inversiones/Activos & $\mathrm{I} 3$ & $\begin{array}{l}\text { Disponibilidades/Total Captaciones } \\
+ \text { Oblig. Con Costo }\end{array}$ & I18 \\
\hline Activos Fijos/Activos & I4 & $\begin{array}{l}\text { Disponib.+ Inversiones en Depósitos } \\
\text { y Valores/Total Activos }\end{array}$ & I19 \\
\hline $\begin{array}{l}\text { Bienes Recibidos en Recuperación } \\
\text { de Créditos/Activos }\end{array}$ & I5 & $\begin{array}{l}\text { Activos Productivos/Total } \\
\text { Captaciones + Oblig. Con Costo }\end{array}$ & $\mathrm{I} 20$ \\
\hline Otros Activos/Activos & I6 & $\begin{array}{l}\text { Total Gastos grales. y Admtivos/ } \\
\text { Total Captaciones }\end{array}$ & $\mathrm{I} 21$ \\
\hline $\begin{array}{l}\text { Cartera de Créditos Vigentes + } \\
\text { Inver./Activos }\end{array}$ & I7 & $\begin{array}{l}\text { Gasto de Personal/Total Gastos } \\
\text { grales. y Administrativos }\end{array}$ & $\mathrm{I} 22$ \\
\hline $\begin{array}{l}\text { Total Captaciones/Pasivos + } \\
\text { Patrimonio }\end{array}$ & I8 & $\begin{array}{l}\text { Otros Gastos Admtivos/Total Gastos } \\
\text { grales. y Admtivos }\end{array}$ & $\mathrm{I} 23$ \\
\hline Otros Pasivos/Pasivos + Patrimonio & I9 & $\begin{array}{l}\text { Total Gastos grales. y Admtivos/ } \\
\text { Total Gastos }\end{array}$ & $\mathrm{I} 24$ \\
\hline $\begin{array}{l}\text { Capital en Circulación + Reserva } \\
\text { Legal/Pasivos + Patrimonio }\end{array}$ & $\mathrm{I} 10$ & Activos Productivos/Total Pasivos & $\mathrm{I} 25$ \\
\hline Patrimonio/Pasivos + Patrimonio & I11 & $\begin{array}{l}\text { Rendimiento Financiero/Activos } \\
\text { Productivos }\end{array}$ & $\mathrm{I} 26$ \\
\hline $\begin{array}{l}\text { Cartera de Créditos Vigentes/Cartera } \\
\text { de Créditos }\end{array}$ & $\mathrm{I} 12$ & $\begin{array}{l}\text { Gastos Financieros/Total } \\
\text { Captaciones + Oblig. Con Costo }\end{array}$ & $\mathrm{I} 27$ \\
\hline $\begin{array}{l}\text { Cartera de Créditos Vigentes M/N/ } \\
\text { Cartera de Créditos }\end{array}$ & I13 & $\begin{array}{l}\text { Total Gastos grales. y Admtivos / } \\
\text { Total Captaciones + Oblig. Con } \\
\text { Costo }\end{array}$ & $\mathrm{I} 28$ \\
\hline $\begin{array}{l}\text { Cartera de Créditos Vigentes M/E/ } \\
\text { Cartera de Créditos }\end{array}$ & I14 & Rentabilidad Patrimonial & I 29 \\
\hline $\begin{array}{l}\text { Cartera de Créditos Vencidos/Cartera } \\
\text { de Créditos }\end{array}$ & I15 & Contingencia/Activos & $\mathrm{I} 30$ \\
\hline
\end{tabular}




\section{Anexo 2: ALGUNOS GRÁFICOS DE DOS INDICADORES}
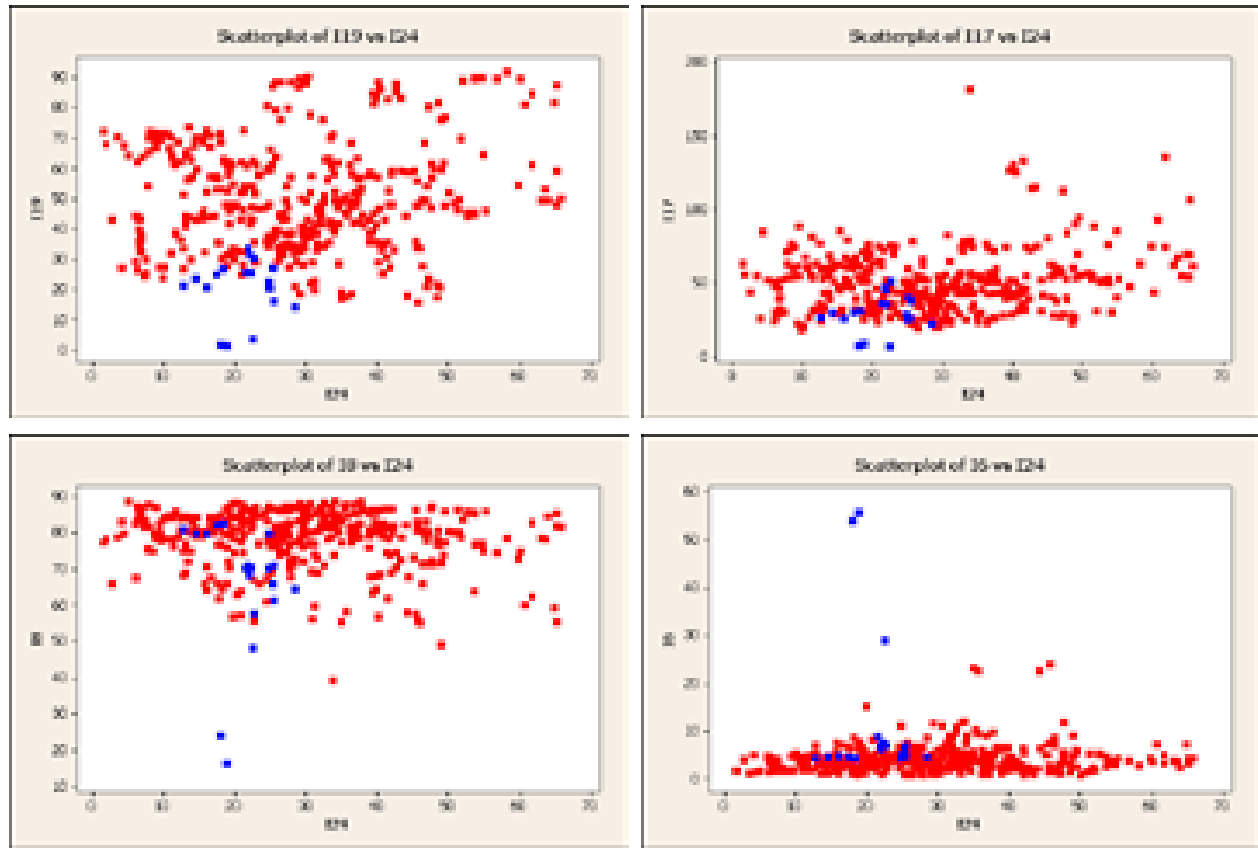

Los puntos rojos son indicadores mensuales de bancos sin problema, los puntos azules son de bancos fallidos o en problemas. 


\section{Anexo 3: ALGUNO GRÁFICOS DE TRES INDICADORES}
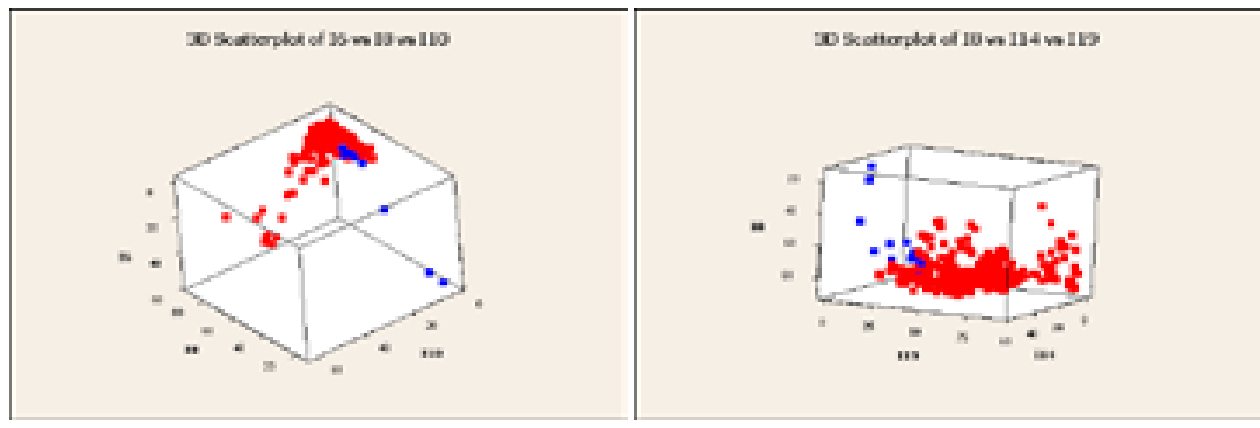

Xosuberplot of $114 \mathrm{n} 117 \mathrm{w} 1 \mathrm{~s}$

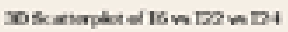
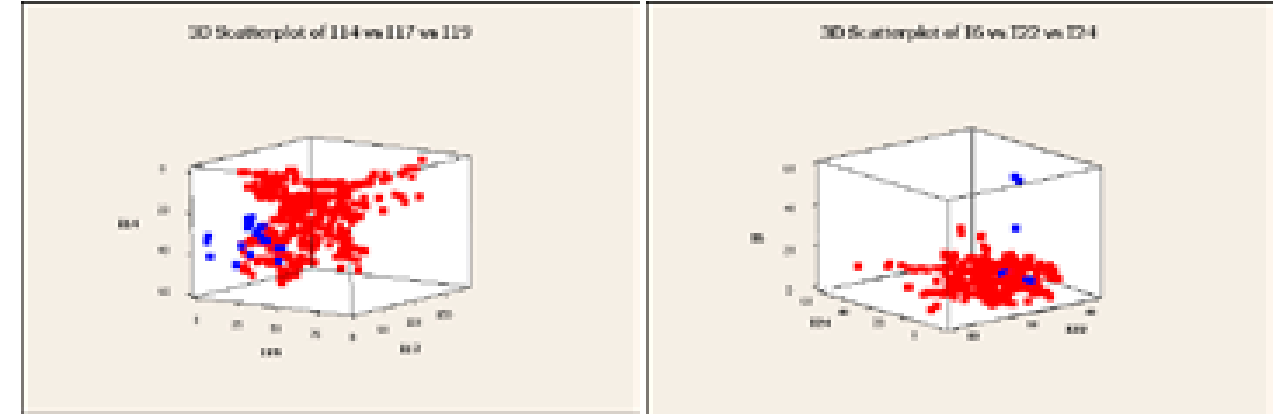

Los puntos rojos son indicadores mensuales de bancos sin problema, los puntos azules son de bancos fallidos o en problemas. 
Anexo 4: MUESTRA DE ALGUNOS EJEMPLOS DE LOS CONJUNTOS DE ENTRENAMIENTO Y PRUEBA

\begin{tabular}{|c|c|c|c|c|c|c|c|c|c|}
\hline \multirow{2}{*}{ Etiqueta } & \multicolumn{9}{|c|}{ INDICADORES } \\
\hline & I6 & I8 & I10 & I14 & I17 & I19 & I20 & I22 & I24 \\
\hline 0 & 5.48 & 56.27 & 5.23 & 29.61 & 35.00 & 28.72 & 107.86 & 47.79 & 30.86 \\
\hline 0 & 2.81 & 76.71 & 7.90 & 33.82 & 26.76 & 20.53 & 81.76 & 37.11 & 48.81 \\
\hline 0 & 7.43 & 73.89 & 9.01 & 41.38 & 23.10 & 22.83 & 80.02 & 36.13 & 31.26 \\
\hline 1 & 6.23 & 65.90 & 7.86 & 33.61 & 40.34 & 27.12 & 71.53 & 43.79 & 25.29 \\
\hline 1 & 4.78 & 79.66 & 7.40 & 22.70 & 25.38 & 20.34 & 79.03 & 43.95 & 25.01 \\
\hline 0 & 4.82 & 77.24 & 6.23 & 46.07 & 23.92 & 19.08 & 82.61 & 46.43 & 35.04 \\
\hline 1 & 4.30 & 70.13 & 8.59 & 40.27 & 29.01 & 20.48 & 76.45 & 45.69 & 24.83 \\
\hline 0 & 2.09 & 79.10 & 12.86 & 23.95 & 62.23 & 49.22 & 48.82 & 45.40 & 27.15 \\
\hline 0 & 22.54 & 58.33 & 48.22 & 0.00 & 29.69 & 17.32 & 95.82 & 46.56 & 35.51 \\
\hline 0 & 4.09 & 81.56 & 9.92 & 0.84 & 29.89 & 25.15 & 82.91 & 58.38 & 7.46 \\
\hline 0 & 2.07 & 86.20 & 8.88 & 9.82 & 33.74 & 29.09 & 73.51 & 63.46 & 22.70 \\
\hline 0 & 9.10 & 49.11 & 47.47 & 0.00 & 90.04 & 75.87 & 68.74 & 36.37 & 49.06 \\
\hline 0 & 2.09 & 59.66 & 3.45 & 13.89 & 64.19 & 41.36 & 72.66 & 69.18 & 31.13 \\
\hline 0 & 1.09 & 79.48 & 6.37 & 12.44 & 38.58 & 30.84 & 76.85 & 56.16 & 19.24 \\
\hline 0 & 5.76 & 56.82 & 4.58 & 33.08 & 34.42 & 33.36 & 109.05 & 50.14 & 19.75 \\
\hline 0 & 3.10 & 77.92 & 7.42 & 35.07 & 26.89 & 21.03 & 80.84 & 51.94 & 46.92 \\
\hline 0 & 6.96 & 74.95 & 8.21 & 43.28 & 24.23 & 26.72 & 78.50 & 37.79 & 12.66 \\
\hline 1 & 7.53 & 70.59 & 7.92 & 31.26 & 35.47 & 25.58 & 68.87 & 46.04 & 22.23 \\
\hline 1 & 4.20 & 80.98 & 7.05 & 25.22 & 26.21 & 21.43 & 77.05 & 42.71 & 12.67 \\
\hline 0 & 4.91 & 78.25 & 6.01 & 47.26 & 22.74 & 18.39 & 83.17 & 51.61 & 29.29 \\
\hline 1 & 4.41 & 64.58 & 8.78 & 45.45 & 21.96 & 14.33 & 83.27 & 43.92 & 28.53 \\
\hline 0 & 2.11 & 75.61 & 11.59 & 26.86 & 71.39 & 53.97 & 46.28 & 43.24 & 7.73 \\
\hline 0 & 22.48 & 58.04 & 49.55 & 0.00 & 30.53 & 17.72 & 96.01 & 48.01 & 44.14 \\
\hline 0 & 3.60 & 83.56 & 9.96 & 0.93 & 30.44 & 26.22 & 80.05 & 60.61 & 6.18 \\
\hline 0 & 2.33 & 83.90 & 9.57 & 5.81 & 29.65 & 24.88 & 78.52 & 64.41 & 20.97 \\
\hline 0 & 7.29 & 59.94 & 38.21 & 0.00 & 92.80 & 81.10 & 45.23 & 62.36 & 60.73 \\
\hline 0 & 2.61 & 68.99 & 3.86 & 15.41 & 46.90 & 35.18 & 69.35 & 67.63 & 31.60 \\
\hline 0 & 2.42 & 79.97 & 6.49 & 9.72 & 38.98 & 31.36 & 74.07 & 52.89 & 26.75 \\
\hline 0 & 4.68 & 57.05 & 4.90 & 31.55 & 36.85 & 32.34 & 106.12 & 52.27 & 20.82 \\
\hline 0 & 3.14 & 78.33 & 7.56 & 32.68 & 27.69 & 21.77 & 80.00 & 44.11 & 48.39 \\
\hline 0 & 7.59 & 75.80 & 8.01 & 42.00 & 24.94 & 27.63 & 77.17 & 36.32 & 18.77 \\
\hline 1 & 8.82 & 70.15 & 8.17 & 33.50 & 35.92 & 25.75 & 66.79 & 48.18 & 21.45 \\
\hline 1 & 4.67 & 79.71 & 7.07 & 26.32 & 25.99 & 20.64 & 77.40 & 41.63 & 16.03 \\
\hline 0 & 5.38 & 77.01 & 5.93 & 48.16 & 25.20 & 19.91 & 82.53 & 50.06 & 31.22 \\
\hline 0 & 1.72 & 75.97 & 10.29 & 33.79 & 81.68 & 62.05 & 37.68 & 43.23 & 11.42 \\
\hline 0 & 23.96 & 55.85 & 51.38 & 0.00 & 27.92 & 15.60 & 100.60 & 47.37 & 45.77 \\
\hline 0 & 3.19 & 83.55 & 9.36 & 0.98 & 39.40 & 32.97 & 71.67 & 60.35 & 6.67 \\
\hline 0 & 2.37 & 84.23 & 8.78 & 8.14 & 35.47 & 29.88 & 71.91 & 58.68 & 19.84 \\
\hline 0 & 7.21 & 59.16 & 36.38 & 0.00 & 69.55 & 81.57 & 70.95 & 70.71 & 64.88 \\
\hline 0 & 2.23 & 70.97 & 3.90 & 20.41 & 44.26 & 34.26 & 70.21 & 66.56 & 32.60 \\
\hline 0 & 1.21 & 79.20 & 8.02 & 11.92 & 43.26 & 34.43 & 73.50 & 56.87 & 27.59 \\
\hline 0 & 4.61 & 55.67 & 4.89 & 33.16 & 47.35 & 35.58 & 100.63 & 53.33 & 22.67 \\
\hline 0 & 2.82 & 80.10 & 8.38 & 33.76 & 28.85 & 23.54 & 78.66 & 41.67 & 47.21 \\
\hline 0 & 8.17 & 77.59 & 7.52 & 41.53 & 23.25 & 32.38 & 77.65 & 35.19 & 20.80 \\
\hline
\end{tabular}

